\title{
Comportements non-linéaires des structures flottantes dans la houle
}

\author{
Non-linear behaviour of structures \\ afloat in waves
}

\author{
B. Molin \\ Ch. Berhault \\ Institut français du pétrole \\ BP 311, 92506 Rueil-Malmaison Cedex
}

\begin{abstract}
Basés sur la théorie dite du potentiel linéarisé, les modèles de diffraction-radiation, dont beaucoup ont été développés en France, permettent de calculer avec une bonne précision les fonctions de transfert de la réponse de structures diverses (navires, plates-formes) dans la houle mais ils ne rendent pas compte de tous les phénomènes qui peuvent être observés expérimentalement (efforts moyens d'ancrage non nuls, oscillations basse fréquence en houle irrégulière, résonances à des fréquences disjointes de celles du spectre de houle, instabilités dynamiques,...), qui, pour être appréhendés, nécessitent des théories et des modèles non-linéaires.
\end{abstract}

Un effort en ce sens a été entrepris à l'Institut français du Pétrole. L'article présente les principaux résultats obtenus.

Based on the theory known as linearized potential, the diffraction-radiation models, many of which have been developed in France, make it possible to accurately calculate the transfer functions of the response of various structures (ships, platforms) in waves, but they do not account for all the phenomena which can be observed (non nil anchorage mean stresses, low-frequency oscillations during irregular waves, resonances with frequencies disconnected from those of the wave spectrum dynamic instabilities...) which in order to be understood, require non-linear models and theories.

An effort in this direction has been made by the French Petroleum Institute (IFP). The article lists the main results obtained.

\section{Introduction}

Le problème abordé ici est celui du mouvement de structures marines de relativement grandes dimensions (navires, semi-submersibles) au point fixe dans la houle.

La dernière décade a vu le développement des modèles numériques dits de diffraction radiation, basés sur la théorie du potentiel linéarisé, qui permettent de construire les fonctions de transfert de la réponse à la houle de la structure considérée. Dans le cadre de cette théorie, le flotteur répond linéairement à la houle, c'est-à-dire que le mouvement prend place aux mêmes pulsations, et que son amplitude est proportionnelle à celle de la houle incidente. Si un tel comportement est confirmé par l'expérience, il est également souvent mis en défaut. Le roulis des navires à la résonance est un exemple bien connu de réponse non linéaire. Les essais en bassin, ou les mesures au réel, montrent par ailleurs que d'autres types de mouvement 
peuvent apparaître, qui se superposent à la réponse linéaire. Ainsi une structure ancrée en houle irrégulière prend un mouvement de dérive lente de grande amplitude dans le plan horizontal, mouvement qui apparaît aux périodes propres du système structure + ancrage. Enfin, dans certains cas, on observe des mouvements "parasites " prenant place à des sub ou super harmoniques de la pulsation de la houle incidente.

Au stade de la conception d'une structure flottante, il est important de pouvoir prédire l'apparition de tels types de comportement et d'en évaluer l'importance. Une action de recherche en ce sens a été initiée il y a quelques années à l'I.F.P., et on en décrit ici sommairement les résultats principaux.

Dans un premier temps, on procède à un bref rappel de la théorie linéaire, et des hypothèses sur lesquelles elle est fondée. Puis, on distingue deux types de non-linéarités dans la réponse du flotteur : non-linéarités de chargement et non-linéarités mécaniques. Pour chaque type on fait le bilan des résultats obtenus et des implications au niveau de la connaissance du comportement des structures flottantes. Pour finir, on insiste particulièrement sur les problèmes qui restent à résoudre.

\section{Réponse linéaire - Fonctions de transfert}

Les hypothèses de base à la théorie dite du potentiel linéarisé, sur laquelle sont fondés les modèles de diffraction-radiation sont les suivantes :

- fluide incompressible, non visqueux;

- écoulement irrotationnel;

- faible cambrure de la houle;

- amplitude de houle et mouvement du flotteur petits devant les dimensions propres du flotteur;

- position moyenne du centre de gravité invariable.

Les deux premières hypothèses permettent de se placer dans la théorie des fluides parfaits et de construire l'écoulement à partir d'un potentiel des vitesses harmonique dans le domaine fluide. Les suivantes permettent de travailler dans le domaine fluide au repos et d'utiliser les cadres de la théorie de la houle infinitésimale et de la théorie des petits mouvements. Formellement on introduit un infiniment petit $\varepsilon$ et on développe toutes les grandeurs entrant en jeu : élévation de surface libre, efforts, mouvements, potentiel etc., sous la forme :

$$
\begin{aligned}
& \eta=\eta^{(0)}+\varepsilon \eta^{(1)}+\varepsilon^{2} \eta^{(2)}+\ldots \\
& \vec{F}=\vec{F}^{(0)}+\varepsilon \vec{F}^{(1)}+\varepsilon^{2} \vec{F}^{(2)}+\ldots \\
& \vec{X}=\vec{X}^{(0)}+\varepsilon \vec{X}^{(1)}+\varepsilon^{2} \vec{X}^{(2)}+\ldots \\
& \Phi=\varepsilon \Phi^{(1)}+\varepsilon^{2} \Phi^{(2)}+\ldots
\end{aligned}
$$

Le cas limite $\varepsilon=0$ correspond à l'état de repos.

Il est alors tout naturel de négliger les termes d'ordre en $\varepsilon$ égal ou supérieur à 2 , ce qui permet de linéariser les équations du problème. La linéarité conduit à séparer les variables d'espace et de temps, et donc à supposer la houle incidente régulière de pulsation $\omega$ et d'amplitude (infinitésimale) $a$ (houle d'Airy). Classiquement le problème est décomposé en un problème de diffraction et six problèmes élémentaire de radiation, résolubles numériquement et le mouvement du flotteur est solution de l'équation :

$$
\left[M+M_{a}(\omega)\right] \frac{d^{2} \vec{X}}{d t^{2}}+B(\omega) \frac{d \vec{X}}{d t}+K \vec{X}=\vec{F}
$$

$M$ : matrice des masses inerties propres

$M_{a}:$ matrice des masses inerties ajoutées

$B$ : matrice des amortissements de radiation

$K$ : matrice des raideurs (hydrostatiques + ancrage)

$\vec{F}$ : efforts de diffraction

Efforts et mouvements étant de la forme :

$$
\begin{aligned}
& \vec{F}=a R_{e}\left\{\vec{f} e^{-i \omega t}\right\} \\
& \vec{X}=a R_{c}\left\{\vec{x} e^{-i \omega t}\right\}
\end{aligned}
$$

Pratiquement le problème se ramène donc au calcul des masses et inerties ajoutées, amortissements et efforts de diffraction, ce qui, pour un flotteur de formes quelconques, suppose l'utilisation de modèles numériques élaborés, dits de diffraction-radiation. De tels modèles, dont plusieurs sont disponibles en France, ont été abondamment décrits par ailleurs [1], [2], [3], [4], [5].

Répétant ce calcul pour différentes valeurs de la pulsation $\omega$, il est possible de construire les fonctions de transfert de la réponse qui, en vertu du principe de superposition, peuvent également s'appliquer pour étudier le mouvement du flotteur en houle irrégulière.

Un exemple de comparaison entre résultats numériques et valeurs expérimentales est fourni par la figure 1 , qui montre la réponse en pilonnement d'un tanker pour une houle de trois quarts. On note un bon accord entre valeurs numériques et expérimentales et on vérifie en outre que la fonction de transfert obtenue à partir d'essais en houle irrégulière coïncide bien avec celle obtenue en houle régulière. L'hypothèse de linéarité de la réponse, sur cet exemple, est parfaitement vérifiée.

Un autre exemple est fourni par la figure 2 qui montre la réponse en tangage d'une bouée cylindrique (diamètre $8 \mathrm{~m}$, tirant d'eau $8 \mathrm{~m}$ ) obtenue à partir d'essais en houle régulière. Le pic de droite correspond à la résonance et on constate que la fonction de transfert y est dépendante de l'amplitude de houle, donc que la réponse en tangage est non-linéaire. Les deux pics de gauche enfin ne sont pas prédits par la théorie linéaire, et correspondent à des pulsations de houle $\omega_{1}$ et $\omega_{2}$ telles que :

$$
\begin{gathered}
\omega_{1}=\omega_{t}+\omega_{p} \\
\omega_{2}=2 \omega_{t}
\end{gathered}
$$

$\omega_{t}$ étant la pulsation propre en tangage et $\omega_{p}$, celle en pilonnement.

Dans les deux cas, le mouvement le tangage apparait, non pas à la pulsation de la houle $\omega$, mais à la pulsation propre $\omega_{i}$. Un tel comportement ne peut s'expliquer que par des phénomènes non-linéaires.

On a mentionné que le calcul ou la détermination expérimentale des fonctions de transfert de la réponse en houle régulière permettait d'étudier le mouvement de la structure considérée en houle irrégulière. Au premier ordre d'approximation, en effet, on peut considérer une houle irrégulière comme une superposition infinie de houles d'Airy, du type :

$$
\eta_{l}=\sum_{i} a_{j} \cos \left(k_{j} x-\omega_{i} t+\psi_{j}\right)
$$




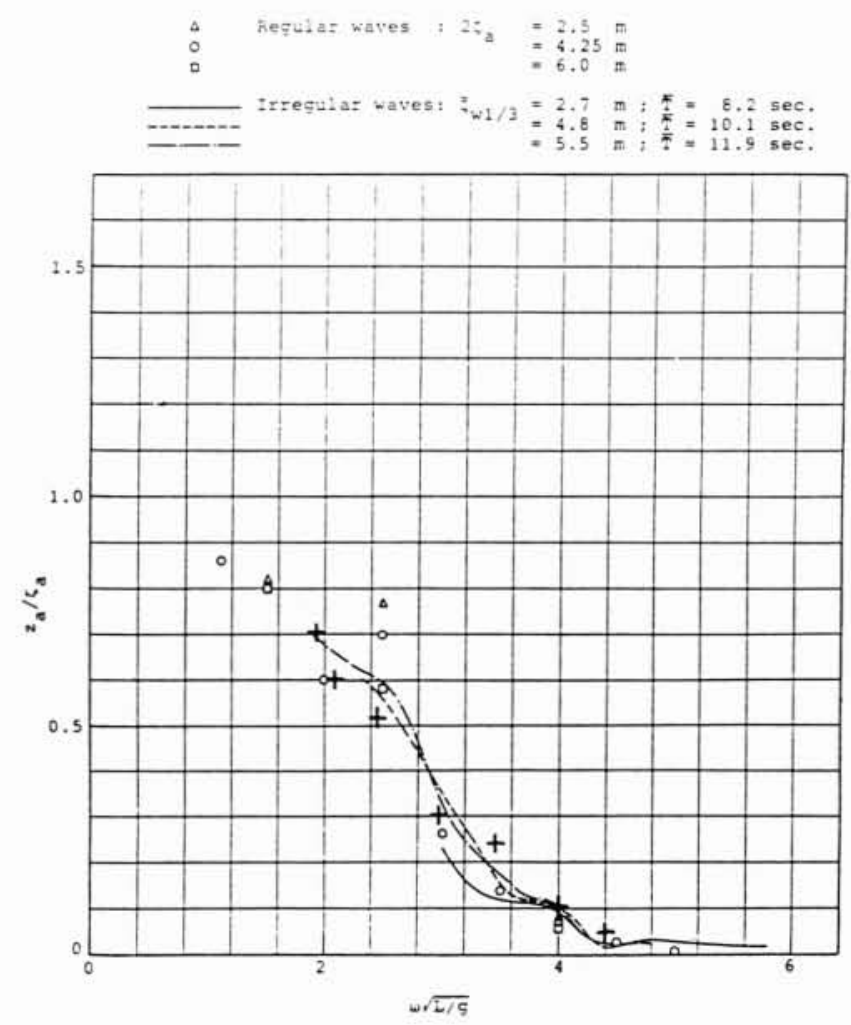

Figure 1. - Réponse au pilonnement d'un tanker. Houle de trois quarts avant.

Remarquer la coïncidence des courbes de transfert obtenues :

- à partir d'essais en houle régulière

- à partir d'essais en houle irrégulière

- par le calcul (signes +).

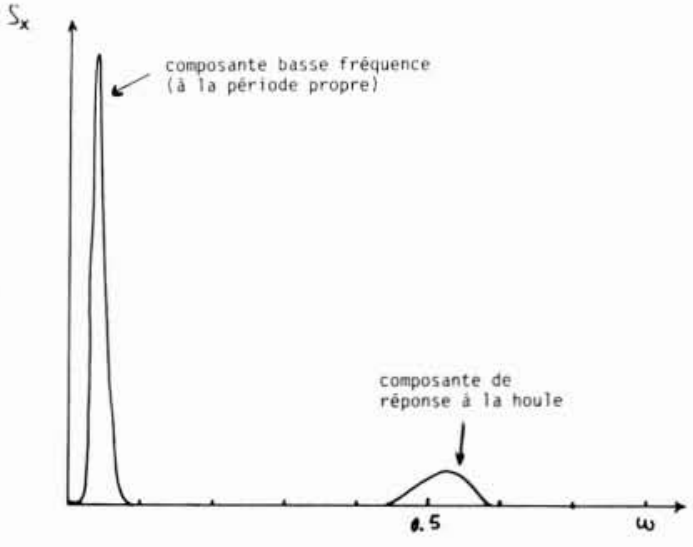

Figure 2. - Fonction de transfert en tangage. On remarque des résonances aux pulsations $\omega_{r} / 2$ et $\omega_{p}+\omega_{r}$ non prédits par la théorie linéaire.

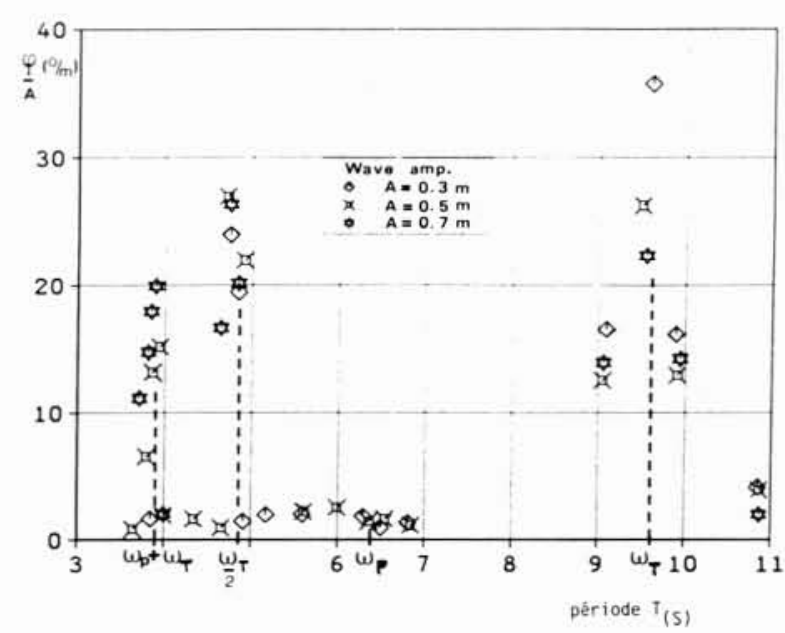

Figure 3, - Spectre du mouvement de cavalement d'un tanker amarré.

\section{où $a_{i}^{2}=2 S\left(\omega_{i}\right) \delta \omega$}

$S(\omega)$ étant le spectre d'énergie de la houle incidente.

La réponse du flotteur à la houle s'écrit alors :

$$
\vec{X}=\sum a_{i} R_{r}\left\{\vec{x}\left(\omega_{i}\right) e^{-r\left(\omega_{i}+\omega_{i}\right)}\right\}
$$

et le spectre de réponse suivant le degré de liberté $k$ se déduit du spectre de houle par:

$$
S x_{k}(\omega)=S(\omega)\left\|x_{k}(\omega)\right\|^{2}
$$

La figure 3 montre un spectre typique de mouvement de cavalement pour un tanker en houle irrégulière. On observe deux pics. Celui de droite, peu énergétique, apparaît aux fréquences de la houle et on peut montrer qu'il est correctement prédit en appliquant (7). On met facilement en évidence que celui de gauche apparaît à la fréquence propre du mouvement de cavalement du tanker, beaucoup plus basse du fait de la faible raideur de l'aussière. Cette composante est beaucoup plus énergétique et c'est elle qu'il convient de considérer pour dimensionner l'ammarrage.

Ces deux exemples illustrent différents types de comportement non-linéaires. Ils ont en commun le fait d'apparaître à des fréquences voisines de la fréquence propre du mouvement considéré, mais les mécanismes d'apparition sont différents.

Dans le cas du tanker, la non-linéarité est entièrement contenue dans le terme d'excitation et on peut montrer que le mouvement de cavalement basse fréquence reste régi par une équation du type :

$$
(M+M a) \ddot{x}+B x=K x=F_{\mathrm{v}}(t)
$$

mais où il convient d'introduire dans $F_{1}(t)$ des termes d'ordre supérieur (du deuxième ordre en $\varepsilon$ ).

Le cas de la bouée est beaucoup plus complexe et conduit à prendre en compte dans l'équation du mouve- 
ment des termes d'amortissement et de raideur non constants. L'introduction dans la raideur d'une fonction sinusoïdale du temps donne à l'équation du mouvement une forme analogue de l'équation de Mathieu, qui explique l'apparition d'un mouvement de tangage à la pulsation moitié de celle de la houle, ou l'apparition simultanée de tangage et de pilonnement à leurs fréquences propres.

On peut évidemment imaginer des situations où le comportement non-linéaire d'une structure flottante relève à la fois de non-linéarités de chargement (le cas du tanker) et de non-linéarités mécaniques (le cas de la bouée). En fait, à bien réfléchir à la complexité du problème du mouvement d'une structure flottante dans la houle, on se convainc aisément que ces types de non-linéarités sont liés. Ils ont été toutefois à la base des deux directions de recherche menées à l'I.F.P. et qui sont décrites ici.

\section{Non-linéarités de chargement}

Le principe est de faire apparaitre dans les efforts hydrodynamiques sur une structure (fixe ou mobile), des termes d'ordre supérieur par rapport à l'amplitude de la houle incidente. Pratiquement, le mieux que l'on sache faire est de poursuivre le type de développement indiqué en (1) à l'ordre suivant : le deuxième ordre.

Le processus de perturbation utilisé entraîne que les termes de deuxième ordre sont des fonctions quadratiques des termes de premier ordre. On en déduit, au niveau des efforts, une dépendance différente en fonction de la variable temps.

\subsection{Houle régulière}

Ainsi, si en houle régulière les efforts excitateurs du premier ordre s'écrivent :

$$
\vec{F}^{(1)}=a R_{\varepsilon}\left\{\vec{f}^{(1)} e^{-\imath e}\right\}
$$

au deuxième ordre, ils prennent la forme :

$$
\vec{F}^{(2)}=a^{2} \vec{f}_{0}^{(2)}+a^{2} R_{c}\left\{\vec{f}_{2}^{(2)} e^{-2(e)}\right\}
$$

On fait donc apparaitre des efforts proportionnels au carré de l'amplitude de la houle incidente, dont une composante, les efforts de dérive, ne dépend plus de la variable temps et la seconde est périodique à la pulsation double.

Pratiquement les efforts de dérive sont repris par le système d'ancrage et il est donc particulièrement important d'y avoir accès.

On montre facilement [6] que leur calcul ne requiert que la connaissance de l'écoulement au premier ordre et peut donc facilement s'effectuer à l'aide des codes linéaires de diffraction/radiation. Un exemple est fourni par la figure 4 qui montre les efforts de dérive en cavalement, calculés et mesurés, pour un tanker par houle de face. Le calcul des efforts de dérive est maintenant effectué de façon standard par la majorité des codes de diffraction/ radiation.

Les efforts de deuxième ordre à la pulsation double $\vec{F}_{2}^{|2|}$ sont plus complexes à appréhender, car ils dépendent de la composante de deuxième ordre $\Phi^{(2)}$ du potentiel $\Phi$ de l'écoulement. Celle-ci obéit à un système d'équation aux limites similaires à celui vérifié par la composante du premier ordre $\Phi^{(1)}$, à la différence près que la condition de surface libre est non homogène. Il s'ensuit quelques complications, au niveau de l'analyse théorique et de la résolution numérique, sur lesquelles ont buté plus d'un. Récemment à l'I.F.P. un modèle numérique a été développé qui donne accès aux efforts et mouvements de deuxième ordre à la pulsation double, pour des flotteurs à révolution verticale [7]. Des exemples d'utilisation sont portés sur les figures 5 et 6 .

\subsection{Houle irrégulière}

Pour une houle incidente irrégulière d'expression (5), il est facile d'établir que les efforts de deuxième ordre associés ont pour expression très générale :

$$
\begin{aligned}
& \vec{F}^{(2)}=\sum_{i} \sum_{j} a_{i} a_{j} R_{i}\left\{\vec{f}_{-}^{(2)}\left(\omega_{i}, \omega_{j}\right)\right. \\
& \left.\exp \left(i\left[-\left(\omega_{i}-\omega_{j}\right) t+\left(\psi_{i}-\psi_{j}\right)\right]\right)\right\} \\
& +\sum_{i} \sum_{j} a_{i} a_{j} R_{e}\left\{\vec{f}_{+}^{(2)}\left(\omega_{i}, \omega_{j}\right)\right. \\
& \left.\exp \left(i\left[-\left(\omega_{i}+\omega_{j}\right) t+\left(\psi_{i}+\psi_{j}\right)\right]\right)\right\}
\end{aligned}
$$

Ces efforts affectent donc deux domaines de fréquence, situés de part et d'autre des fréquences du spectre de houle (fig. 7). Les fréquences propres des structures flottantes étant généralement assez basses, la composante haute fréquence est de peu d'intérêt (sauf toutefois en ce qui concerne l'excitation en roulis, tangage, pilonnement des plates-formes sur lignes tendues).

Par contre, on met facilement en évidence que la composante basse fréquence, bien que du deuxième ordre, peut exciter de façon significative les structures flottantes à leurs périodes propres. A ces longues périodes, les amortissements de radiation sont quasiment nuls et une faible excitation peut entrainer un mouvement d'amplitude importante. Le cas du mouvement horizontal des navires amarrés est le plus connu [8] mais le même phénomène se retrouve pour les mouvements de roulis, tangage, pilonnement des grosses semi-submersibles ou des réservoirs SPAR [9] (fig. 8).

La détermination exacte de $\vec{f}_{-}^{(2)}\left(\omega_{i}, \omega_{j}\right)$ est du même ordre de difficulté que celle de $\tilde{f}_{2}^{12}$ en houle régulière, à la différence près qu'il conviendrait de l'effectuer pour tous les couples $\left(i, \omega_{j}\right)$. Aussi préfère-t-on en général recourir à des approximations, en tirant parti du fait que :

$$
\lim _{\left|\omega_{i}-\omega_{j}\right| \rightarrow 0} \vec{f}_{-}^{(2)}\left(\omega_{i}, \omega_{i}\right)=\vec{f}_{0}^{(2)}\left(\omega_{i}\right)
$$

et en supposant que la structure considérée ne dépend qu'à très basse fréquence. On utilise alors une expression du type :

$\vec{F}_{-}^{(2)}=\sum_{i} \sum_{j} a_{i} a_{j} \vec{f}_{0}^{(2)}\left(\omega_{i}\right) \cos \left[\left(\omega_{i}-\omega_{j}\right) t-\left(\psi_{i}-\psi_{0}\right)\right]$

qui est équivalente à (10) aux très basses fréquences.

De (10) ou (11), on peut également déduire le spectre des efforts excitateurs à basse fréquence, et en tirer le spectre de réponse à partir de (8). Généralement toutefois, de nombreuses autres non-linéarités entrant en jeu dans le comportement de dérive lente d'une structure amarrée (rappel de l'ancrage, efforts dus au courant, mouvement 


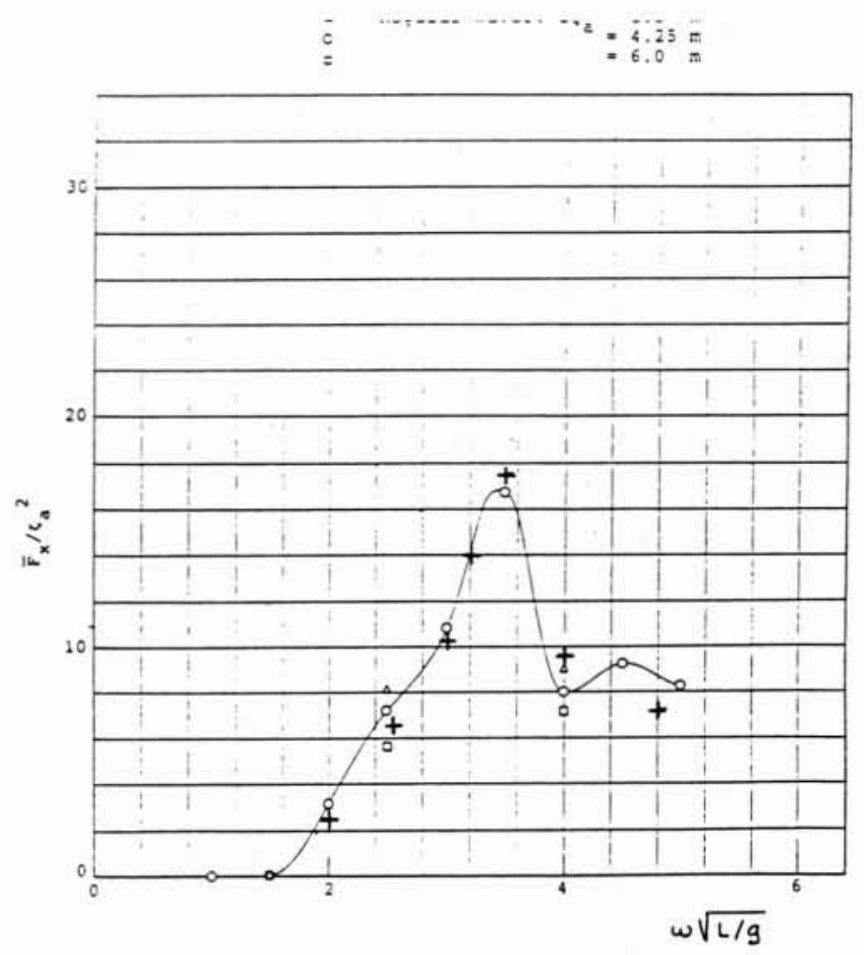

Figure 4. - Efforts de dérive sur un tanker. Force longitudinale - Incidence $0^{\circ}$.

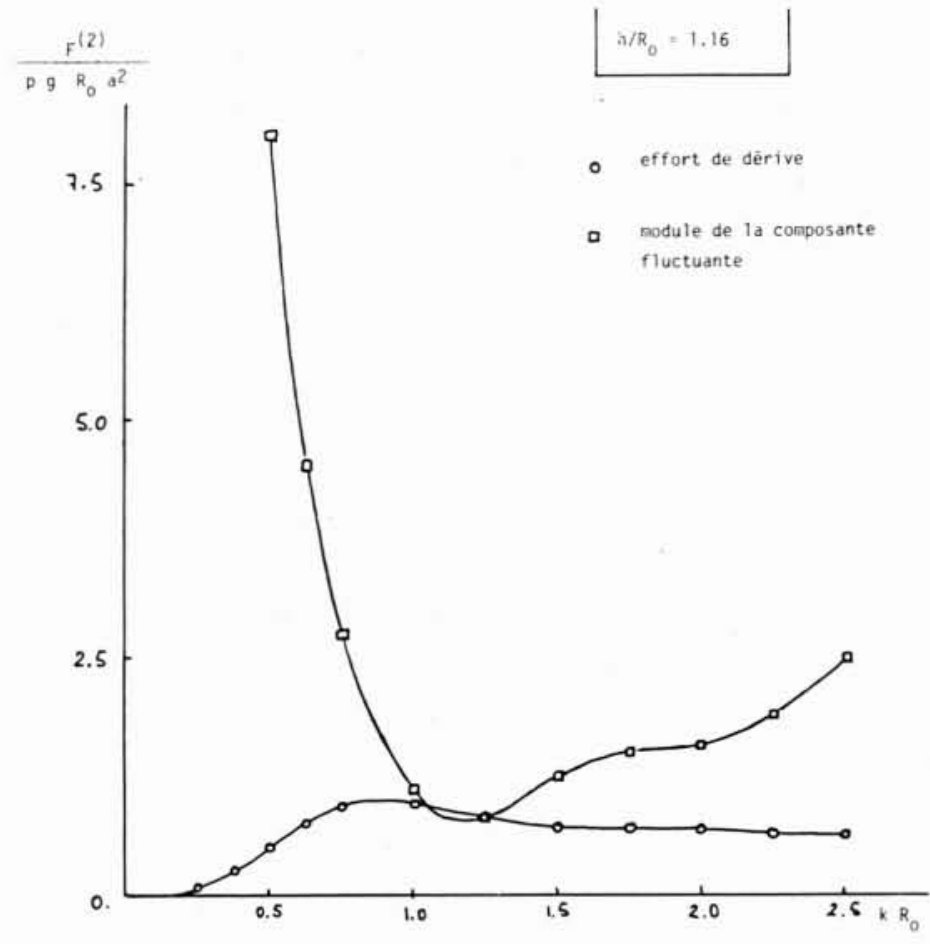

Figure 5. - Effort horizontal de deuxième ordre sur un cylindre vertical.

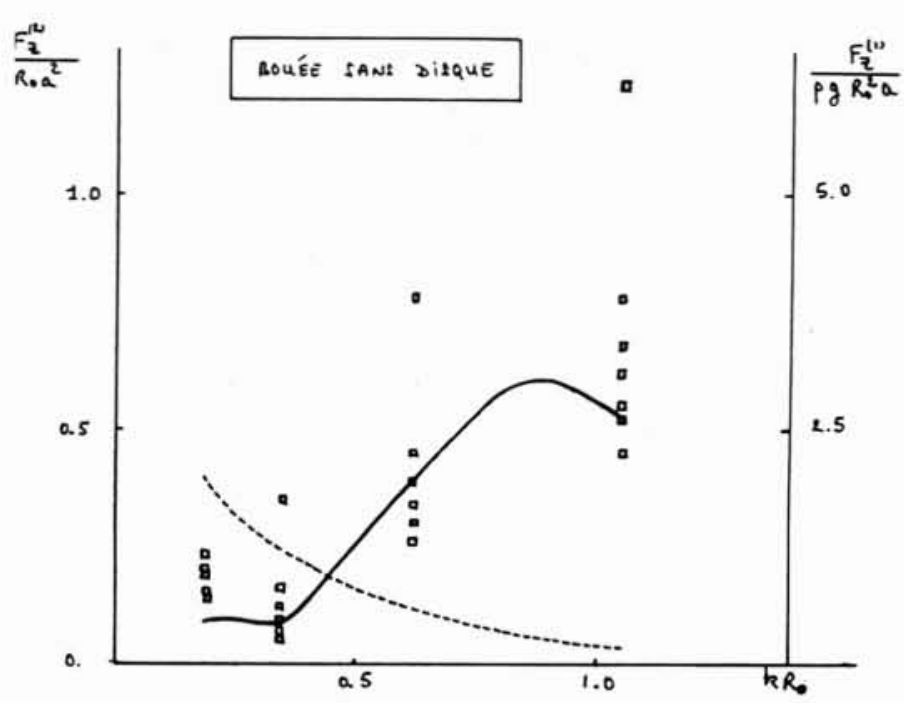

Figure 6. - Diffraction sur une bouée. Modules des efforts verticaux de premier et deuxième ordre.
$\square$ expérimental
- COREV
$2^{\circ}$ ordre
... COREV
$1^{\text {et }}$ ordre
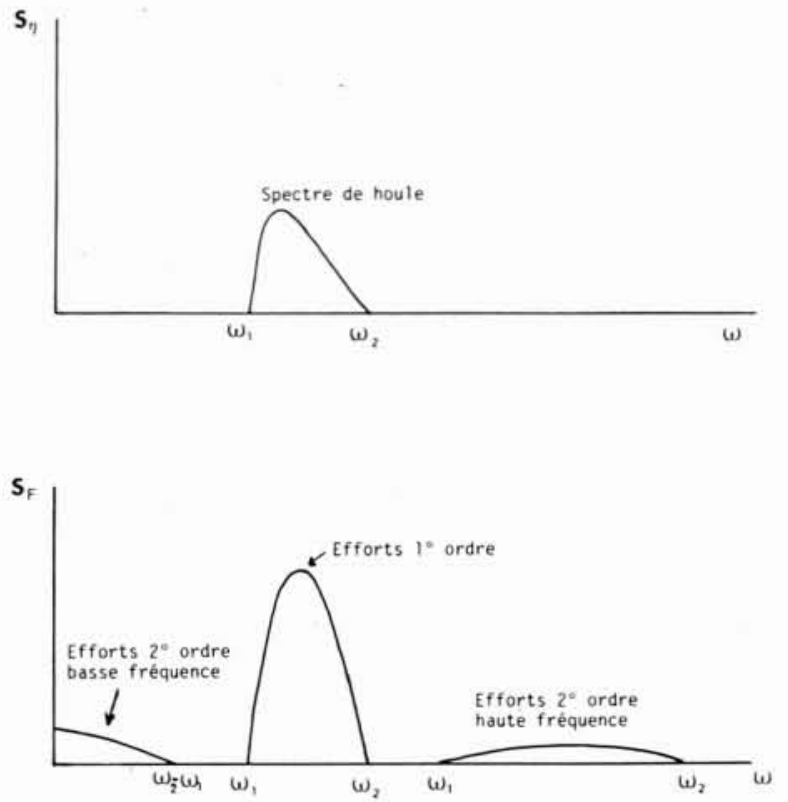

Figure 7. - Spectre des efforts de premier et de deuxième ordres.
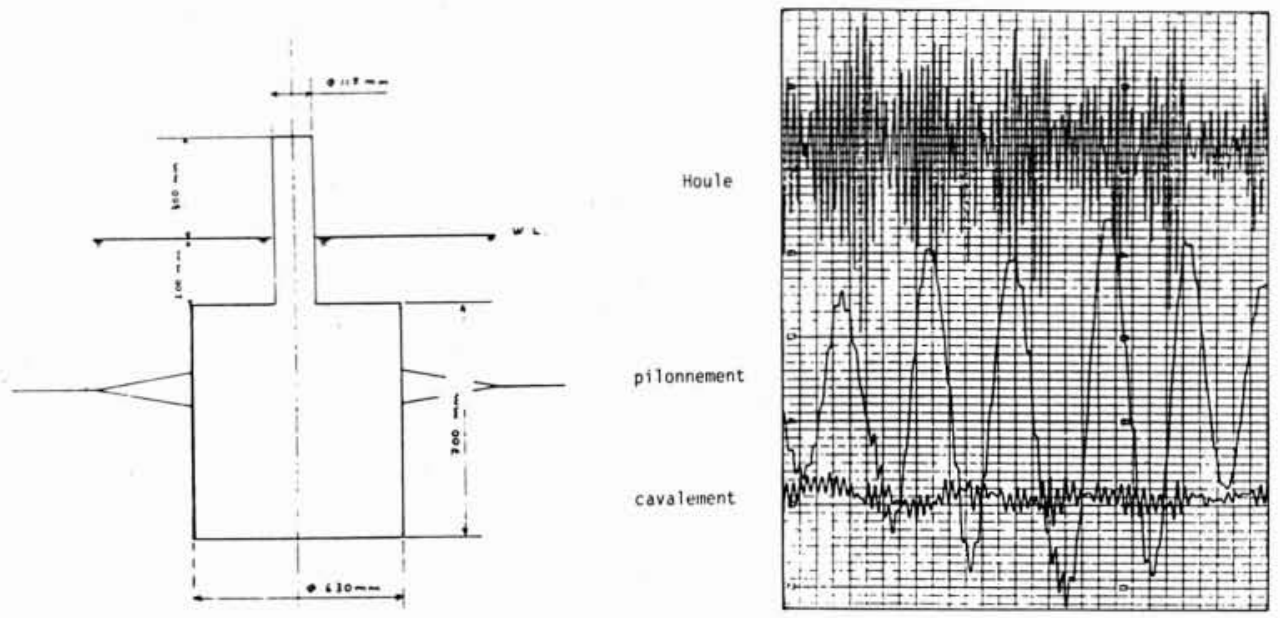

Figure 8. - Réservoir flottant. Mouvements de cavalement et de pilonnement obtenus expérimentalement. Noter l'importance de la composante basse-fréquence (à la fréquence propre) pour le pilonnement. 
de lacet important), on préfère recourir à des modèles de simulation temporelle [8]. Un exemple d'une telle simulation est montré sur la figure 9.
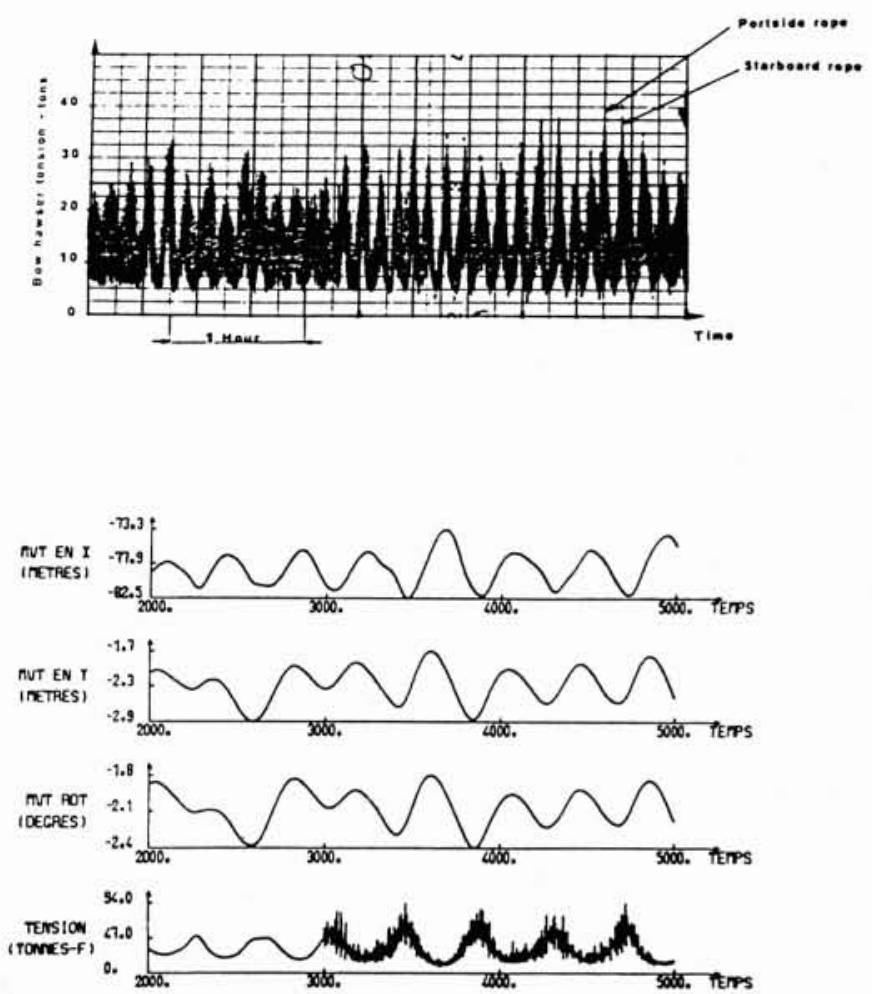

Figure 9. - Comportement d'un tanker amarré à une bouée de chargement. Enregistrement expérimental de la tension et résultats de la simulation numérique (modèle TABUO).

\section{Non-linéarités mécaniques}

Dans le paragraphe précédent, les non-linéarités sont introduites dans les efforts excitateurs. Une forme plus réaliste, bien qu'arbitraire, de l'équation (2) du mouvement est :

$\left(M+M_{a}\right) \frac{d^{2} \vec{X}}{d t^{2}}+B(\vec{X}, \dot{\vec{X}}) \frac{d \vec{X}}{d \gamma}+K(\vec{X}, t) \vec{X}=\vec{F}(t)$

Il est en effet nécessaire de tenir compte des nonlinéarités des termes de raideur et d'amortissement dès que l'amplitude du mouvement devient appréciable.

Les non-linéarités de raideur peuvent provenir :

- des couplages hydrostatiques entre les différents degrés de liberté (roulis-pilonnement par exemple);

- des variations de la raideur de l'ancrage;

- des couplages entre les mouvements et les efforts de diffraction au $2^{\mathrm{e}}$ ordre.

La forme de l'amortissement dépend, quant à elle, de la géométrie de la carène immergée et du régime de l'écoulement.
L'excitation étant supposée harmonique, différents types de réponse peuvent être déduits suivant que la pulsation de l'excitation coïncide ou non avec une pulsation propre du système.

\subsection{Excitation à une fréquence propre}

C'est par exemple le cas bien connu du roulis des navires à la résonance. Il est d'usage, dans son analyse, d'introduire des non-linéarités dans le terme d'amortissement (du type $\dot{\theta}^{3}$ ou $\left.|\dot{\theta}| \dot{\theta}\right)$ et dans le terme de rappel. L'effet de ces non-linéarités est de borner la réponse à la résonance, mais aussi éventuellement de générer des sub et des superharmoniques [10]. Un autre phénomène lié à l'existence d'un rappel non-linéaire est l'apparition de sauts d'énergie dans la réponse, plusieurs niveaux de réponse pouvant coexister pour une même excitation. Un tel phénomène a été notamment rapporté par S.N. Smith, lors de l'étude de la réponse en pilonnement d'un navire de type SWATH [11].

\subsection{Résonances paramétriques}

Des mouvements résonnants peuvent être observés sans qu'il y ait d'excitation à l'une des fréquences propres. $\mathrm{Ce}$ phénomène est lié à la forme du terme de raideur et se produit quand des relations particulières existent entre la fréquence de l'excitation et les fréquences propres des différents degrés de liberté. Un exemple peut être donné par l'équation du type :

$$
\left(M+M_{a}\right) \ddot{X}+B \dot{X}+K(X) X=F(t)
$$

avec

$$
K(X)=K_{0}+K_{1} X
$$

Soit $X_{1}$ la solution de l'équation linéarisée.

$$
\left(M+M_{a}\right) \ddot{X}_{1}+B \dot{X}_{1}+K_{0} X_{1}=F(t)
$$

Une meilleure approximation $X_{2}$ de la solution de (13) peut être obtenue en réinjectant $X_{1}$ dans (13) :

$$
\left(M+M_{a}\right) \ddot{X}_{2}+B \dot{X}_{2}+\left(K_{0}+K_{1} X_{1}\right) X_{2}=F(t)
$$

$f$ et $X_{1}$ sont des expressions harmoniques du temps, on reconnaît alors dans (16) une équation de Mathieu pour laquelle on montre qu'il existe des solutions instables pour des combinaisons particulières de la pulsation excitatrice $\omega$ et de la pulsation propre $\Omega$.

Une étude de ce type d'instabilité a été entreprise à l'I.F.P., dans le cadre d'une thèse de Docteur-Ingénieur [12], sur un cas particulier : celui d'une bouée circulaire (diamètre $=$ tirant d'eau $=8 \mathrm{~m}$ ) pour laquelle de telles instabilités ont été rencontrées expérimentalement.

L'instabilité principale rencontrée concerne le mouvement de roulis qui, bien que non sollicité dans le cadre d'une théorie linéarisée, apparaît à la pulsation moitié de celle de la houle (roulis hémichrome) dans un certain domaine de fréquences, allant de la fréquence propre en pilonnement à deux fois la fréquence propre en roulis. On note sur la figure 10 que les amplitudes de roulis obtenues expérimentalement sont loin d'être négligeables. Une caractéristique importante de cette résonance paramétrique est qu'elle n'apparaît qu'au delà d'un certain seuil de l'excitation, donc d'une certaine amplitude de houle. 
Au cours de l'investigation en référence, d'autres instabilités ont été mises en évidence, et un modèle de simulation a été développé, qui a permis de retrouver, numériquement, le comportement observé. Ce modèle en particulier tient compte de la variation des efforts excitateurs et du rappel hydrostatique avec le mouvement du flotteur [13].

Une question subsidiaire est la possibilité d'apparition de telles instabilités en houle irrégulière. Les simulations numériques effectuées montrent que cette éventualité n'est pas à exclure (figure 11) [14]. Le roulis rythmique des voiliers et des navires de pêche en est d'ailleurs un exemple connu.

Une prochaine étape est de généraliser le modèle développé à l'étude du mouvement d'un flotteur de forme quelconque, ou tout du moins, de pouvoir en prédire les risques d'instabilité. Notons que ce risque d'instabilité est fortement associé à la répartition des périodes propres en pilonnement, roulis et tangage. Une période propre en pilonnement égale à la moitié de celle en roulis ou en tangage est une situation propice à l'apparition de résonances paramétriques.

\section{Conclusion}

Cet exposé avait pour but de sensibiliser le lecteur au problème des comportements non-linéaires des structures flottantes qui peuvent se rencontrer dans les applications pétrolières offshore. Différents types de mécanismes ont été identifiés, qui peuvent générer des mouvements nonlinéaires d'amplitudes bien supérieures à celles prédites dans le cadre de théories linéaires.

Un problème essentiel cependant n'a pas été abordé, qui est celui des amortissements d'origine visqueuse. Tous les mouvements non-linéaires identifiés étant de type résonnant, le calcul de leur amplitude requiert la connaissance des mécanismes de dissipation d'énergie : radiation et effets visqueux, ces derniers étant généralement prépondérants aux fréquences considérées, mais malheureusement difficiles à quantifier. Il apparaît important d'entreprendre un effort de recherche théorique et expérimentale en ce sens.

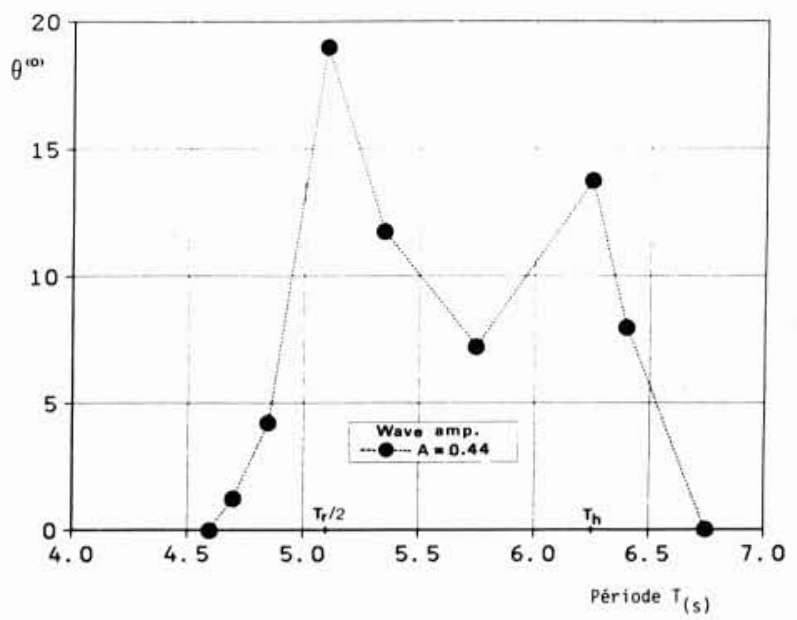

Figure 10. - Pseudo-fonction de transfert en roulis obtenue expérimentalement.

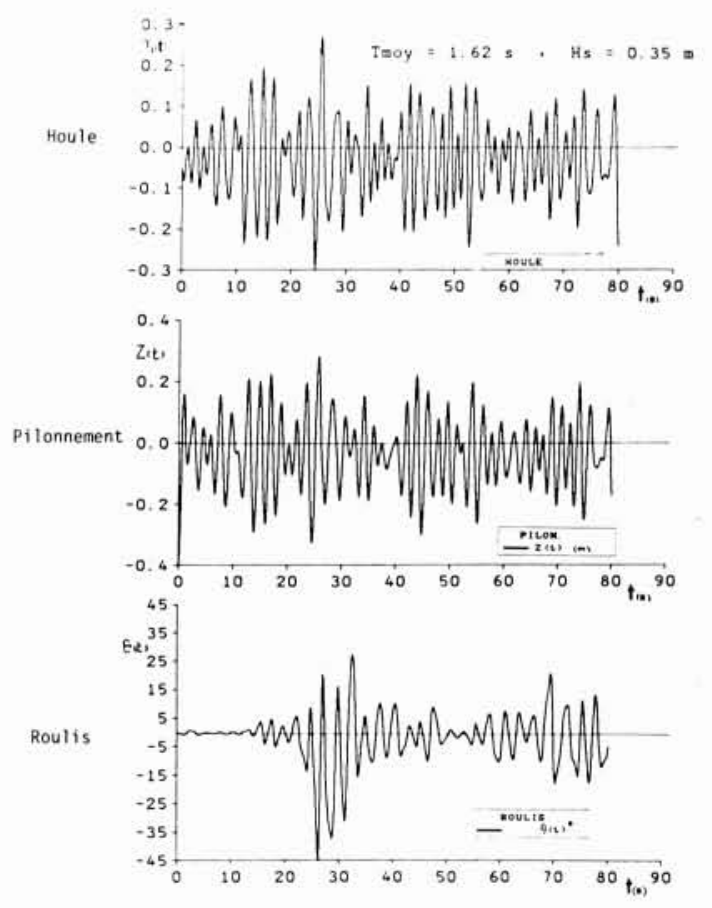

Figure 11, - Résonance paramétrique en houle irrégulière. Résultats d'une simulation numérique. 


\section{Références}

[1] P. Guevel, J.C. Daubisse, G. Delhommeau. - Oscillations des corps flottants soumis aux actions de la houle. ATMA $n^{\circ} 78,1978$.

[2] J.L. Chenot. - Méthode numérique de calcul du mouvement d'un corps flottant soumis à l'influence d'une houle périodique en théorie linéaire. Revue de IIIFP, vol. XXX-5, 1975.

[3] A. JAMI, M. LeNoIR. - A variational Formulation for Exterior Problems in Linear Hydrodynamics. Computer Methods in Applied Mechanics and Engineering, vol. 16, 1978.

[4] C. Berhault. - Symmetric BIEM Applied to the Hydrodynamic Diffraction Radiation Problem. BOSS'79 et Int. Symp. on In. Num. Analysis, Montreal, 1980.

[5] G. Susbielles, C. Berhault. - Comparaison des modèles numériques $3 \mathrm{D}$ de diffraction radiation. Revue de l'IFP, vol. XXXIII-4, 1978.

[6] H. Maruo. - The Drift of a Body Floating in Waves. J. of Ship Research, vol. 4, $\mathrm{n}^{\circ}$ 3, 1960.

[7] B. Molin, A. MARION. - Etude au deuxième ordre du comportement des corps flottants en houle régulière. Rapport IFP 33 031, 1985 et congrès OMAE 86 (à paraître).

[8] B. MoLIN. - TABUO, un modèle de comportement des tankers amarrés sur point unique. Thèse de Docteur-ingénieur, ENSM, 1981.

[9] B. Molin, J.P. Hairault. - On second order motion and vertical drift forces for $3 \mathrm{D}$ bodies in regular waves. Int. Workshop on Ship and Platform Motions, Berkeley: 1983 et DEA de J.P. HAIRAULT (ENSM).

[10] A. Lardo, A. Francescutto, R. Naberco, - Deterministic non-linear rolling : a critical review. ATMA 85 .

[11] S.N. SMITH. - Parasitic motions and capsize safety of a SWATH type Ship, OTC 4619, 1983.

[12] J.M. ChANTREL. - Instabilités paramétriques dans le mouvement des corps flottants. Application au cas des bouées de chargement. Thèse de Docteur-ingénieur, ENSM, 1984.

[13] J.M. Chantrel, C. Berhault. - Parametric resonance of C.A.L.M. buoy motions in regular waves. BOSS'85, Delft. 1985.

[14] C. Berhault. - Instabilités paramétriques dans le mouvement des corps flottants en houle irrégulière. Rapport IFP $33078,1985$. 